\title{
Análisis empírico del modelo de Elección Secuencial en humanos
}

\section{Empirical Analysis of the Sequential Election Model in Humans Análise empírica do modelo de Eleição Sequencial em humanos}

\author{
Óscar García-Leal*, Enzo Leandro Rodríguez Macías \\ Universidad de Guadalajara
}

Doi: http://dx.doi.org/10.12804/revistas.urosario.edu.co/apl/a.4869

\section{Resumen}

Los resultados encontrados en situaciones de elección entre animales humanos y no humanos son, en algunos casos, contradictorios. Se han propuesto dos posibles explicaciones: (i) los procesos de elección son diferentes en animales humanos y no humanos o (ii) los procedimientos utilizados para el estudio de la elección en unos y otros no son comparables. Particularmente, en humanos se observan mayores tiempos de respuesta a medida que se incrementa el número de alternativas que constituyen el contexto de elección. Esto ha sido interpretado como evidencia a favor de un proceso de comparación entre las alternativas presentadas. Sin embargo, estudios recientes con estorninos han encontrado resultados opuestos. Para dar razón de estos resultados se ha propuesto el Modelo de Elección Secuencial, que no asume la existencia de un proceso de comparación y permite realizar predicciones concretas en situaciones de elección momento a momento. Se diseñó una tarea equivalente a las empleadas con animales no humanos para su presentación en humanos. Los resultados apoyaron parcialmente las predicciones del Modelo de Elección
Secuencial, si bien no permiten descartar la existencia de un mecanismo de comparación en humanos.

Palabras clave: elección, modelo de elección secuencial, latencia, valoración, proceso de comparación.

\section{fbstract}

The outcomes reported comparing the behavior of human and non-human animals in choice procedures are sometimes contradictory. Two different hypotheses have been stated: (i) that natural selection has favored different choice processes, or (ii) we cannot compare the performance of human and non-human animals in experimental protocols due to the differing particularities of the protocols used in each case. In humans, there is a clear correlation between the number of alternatives that compose the context of choice and the latency of response. Higher latencies are observed as the number of alternatives increases. This evidence has supported the hypothesis that organisms compare alternatives before making choices. Nevertheless, recent results using Sturnus vulgaris as subjects have reported different results. The Sequential Choice Model (SCM) has been

* Correspondencia. Correo electrónico: oscargl@cencar.udg.mx

Cómo citar este artículo: García-Leal, Ó., \& Rodríguez-Macías, E. L. (2018). Análisis empírico del Modelo de Elección Secuencial en humanos. Avances en Psicología Latinoamericana, 36(1), 139-153. doi: http://dx.doi.org/10.12804/revistas. urosario.edu.co/apl/a.4869 
proposed to account for these data. This model does not assume a process of comparison. Even more importantly, it allows for moment-to-moment predictions in situations of choice. We developed an experimental protocol to be used with humans that reproduces the properties of situations of choice usually considered with non-human animals. The results partially support the predictions of the Sequential Choice Model, but it is not possible to discard a comparison mechanism in situations of choice faced by humans.

Keywords: Choice, sequential choice model, latency, valuation, comparison process.

\section{Resumo}

Os resultados encontrados em situações de eleição entre animais humanos e não humanos são em alguns casos contraditórios. Se têm proposto duas possíveis explicações: (i) os processos de eleição são diferentes em animais humanos e não humanos ou (ii) os procedimentos utilizados para o estudo da eleição em uns e outros não são comparáveis. Particularmente, em humanos se observam maiores tempos de resposta à medida que se incrementa o número de alternativas que constituem o contexto de eleição. Isto tem sido interpretado como evidência a favor de um processo de comparação entre as alternativas apresentadas. No entanto, estudos recentes com estorninhos têm encontrado resultados opostos. Para dar razão destes resultados se tem proposto o Modelo de Eleição Sequencial, que não assume a existência de um processo de comparação e permite realizar predições concretas em situações de eleição momento a momento. Desenhou-se uma tarefa equivalente às empregadas com animais não humanos para a sua apresentação em humanos. Os resultados apoiaram parcialmente as predições do Modelo de Eleição Sequencial se bem que não permitem descartar a existência de um mecanismo de comparação em humanos.

Palavras-chave: eleição, modelo de eleição sequencial, latência, valoração, processo de comparação.
La falta de correspondencia en los resultados obtenidos en situaciones de elección entre humanos y animales no humanos es frecuente y ha sido objeto de atención de manera recurrente. Por ejemplo, en situaciones de autocontrol, en las que se presentan simultáneamente dos alternativas de respuesta, una de las cuales entrega una cantidad pequeña de alimento de manera inmediata, y la otra una cantidad mayor de alimento pero de manera demorada, se ha encontrado que los animales no humanos bajo mayor grado de privación muestran preferencia por la alternativa de demora corta o inmediata (Tanno, Kurashima \& Watanabe, 2011), mientras que bajo otras manipulaciones prefieren la alternativa de reforzador grande y demorado (Bradshaw \& Szabadi, 1992; Feeney \& Roberts, 2008), en tanto que los humanos en general muestran mayor preferencia por la alternativa demorada (Logue, Peña-Correal, Rodríguez \& Kabela, 1986; Navarick, 1996). Sin embargo, alteraciones clínicas como el consumo de alcohol, antecedentes en el abuso de sustancias (Kollins, 2003) o algunas variaciones genéticas hereditarias (Mitchell, 2011) inducen preferencia por la opción pequeña e inmediata.

Se han propuesto dos argumentos para dar razón de estos resultados diferenciales. El primero de ellos alude a las diferencias en el tipo de tareas empleadas (Locey, Hackenberg \& Pietras, 2009; Navarik, 2004). En general, los trabajos con humanos emplean un problema de elección con consecuencias hipotéticas (Kahneman \& Tversky, 1979; Navarick, 1998; Tversky \& Kahneman, 1981) y usualmente las elecciones de los participantes conllevan la obtención de puntos intercambiados al final de la sesión por alguna gratificación. En el caso de los estudios que emplean animales no humanos, por el contrario, las consecuencias tienen un efecto inmediato sobre los participantes (e.g., obtención de alimento consumible de manera inmediata). El segundo argumento propuesto alude a la existencia de mecanismos de elección diferentes en animales humanos y no humanos como 
resultado del proceso de selección natural (e. g., diferencias entre especies).

Varios estudios han señalado que cuando se crean condiciones experimentales comparables en situaciones de elección, los resultados diferenciales desaparecen. Esto ha sido reportado tanto en situaciones de autocontrol (Forzano \& Logue, 1994; Navarick, 1996), como en procedimientos de sensibilidad al riesgo (Locey et al., 2009; Pietras \& Hackenberg, 2003).

El tiempo de respuesta se ha constituido en una variable dependiente de uso generalizado que permite realizar inferencias acerca de la cantidad y complejidad de los procesos implicados en la resolución de una tarea, en la medida en que todo procesamiento de información, dado que implica la realización de cómputos, requiere un tiempo para efectuarse. Conforme la tarea resulta más compleja, y en consecuencia la cantidad de cómputos requeridos incrementa, el tiempo de respuesta inevitablemente también lo hace (Kacelnik, Vasconcelos, Monteiro \& Aw, 2011; Luce, 1986; Sigman \& Dehaene, 2011).

En el estudio de la toma de decisiones, particularmente en humanos, el incremento en los tiempos de respuesta conforme aumenta el número de alternativas que conforman el contexto de elección ha sido considerado como evidencia a favor de la existencia de un mecanismo de comparación del valor de las alternativas presentadas, resultando elegida finalmente aquella alternativa con mayor valor atribuido. Este proceso de comparación subyace a la mayor parte de los modelos de decisión (Kacelnik et al., 2011).

Sin embargo, resultados obtenidos con animales, particularmente estorninos, muestran resultados contrarios a los esperados por los modelos comparativos (Kacelnik et al., 2011; Shapiro, Siller \& Kacelnik, 2008), observándose tiempos de respuesta menores en situaciones de elección con dos o más alternativas, que en situaciones en las que cada una de las alternativas se presenta de manera independiente.
Con la finalidad de dar razón de aparentes violaciones de las predicciones relativas a los tiempos de respuesta de los modelos que asumen la existencia de un proceso de comparación de las alternativas presentadas, Shapiro et al. (2008) propusieron el Modelo de Elección Secuencial (SCM por sus siglas en inglés - Sequential Choice Model-), que no presupone la existencia de un mecanismo de comparación cuando se presenta más de una alternativa en una situación de elección. Para ello, el modelo asume que los procesos implicados en la elección, cuando se presentan dos o más alternativas, son una extensión de aquellos que operan cuando una alternativa se presenta de manera aislada. Una bondad añadida del modelo es que permite realizar predicciones momento a momento en relación con la elección considerando las latencias de respuesta asociadas con cada una de las alternativas presentadas.

El modelo ha recibido soporte empírico en varios estudios (Aw, Monteiro, Vasconcelos \& Kacelnik, 2012; Freidin, Aw \& Kacelnik, 2009; Kacelnik \& Bateson, 1997; Mazur, 2010; Vasconcelos, Monteiro, Aw \& Kacelnik, 2010), pero siempre utilizando estorninos como sujetos experimentales.

Con la finalidad de contrastar la conveniencia de aludir a un proceso de comparación en la toma de decisiones en humanos cuando se igualan las características de las tareas utilizadas con animales no humanos, se realizó una réplica no sistemática del trabajo de Shapiro et al. (2008) utilizando humanos como sujetos experimentales. La tarea fue modificada con la intención de establecer una preparación experimental de propiedades comparable a las utilizadas con animales no humanos en situaciones de elección. Para ello, se consideraron preparaciones utilizadas en estudios previos de elección que han reportado resultados semejantes entre humanos y no humanos (Locey et al., 2009; Navarick, 1996). Adicionalmente, este estudio tuvo como finalidad contrastar la capacidad predictiva del Modelo de Elección Secuencial en humanos. 


\section{Método}

\section{Sujetos}

Participaron en el estudio 41 estudiantes de la Licenciatura en Psicología de una Universidad privada de la ciudad de Guadalajara (Jalisco, México). No se consideraron relevantes las variables sexo y edad. Todos los participantes participaron en el estudio de manera voluntaria.

\section{Procedimiento}

El procedimiento utilizado fue una modificación del descrito en Shapiro et al. (2008) con estorninos.

Cada participante fue expuesto en una primera fase (figura 1) a una tarea de ordenamiento de 4 videos en función de su preferencia. Los videos fueron documentales de libre acceso en español descargados de Youtube con una duración aproximada de 10 minutos. Los contenidos fueron los siguientes: apicultura, inteligencia animal, caricatura de los Simpson y resolución de integrales.

Para la exposición a estos videos se desarrolló una aplicación informática en la que se informaba a los participantes del contenido de cada video y se les permitía visualizar cada uno de ellos durante 40 segundos. Los participantes tenían que ver cada fragmento de video antes de ordenarlos en función de su preferencia. Finalizada la fase de ordenamiento se daba inicio a la fase experimental. En esta fase se presentaron únicamente fragmentos del video de mayor preferencia para cada participante.

Se conformaron cuatro grupos, cada uno de ellos integrado por, aproximadamente, 10 participantes (tabla 1). Cada grupo estuvo definido por la diferencia de razones tiempo de exposición al video - demora (T-D a partir de este momento) entre las dos alternativas (A y B) de elección presentadas. Para el grupo 1 ambas alternativas de respuesta tenían la misma razón T-D $(\mathrm{A}=\mathrm{B})$; para el grupo 2 la razón correspondiente a la alternativa $A$ era dos veces superior a la de la alternativa $\mathrm{B}(\mathrm{A}=2 \mathrm{~B})$; para los grupos 3 y 4 la razón de la alternativa $\mathrm{A}$ era 3 y 4 veces superior, respectivamente, a la de la alternativa $B(3: A=3 B ; 4: A=4 B)$. Para cada grupo se definieron diferentes tratamientos en función de los valores de T-D correspondientes a cada una de las alternativas, tal y como se muestra
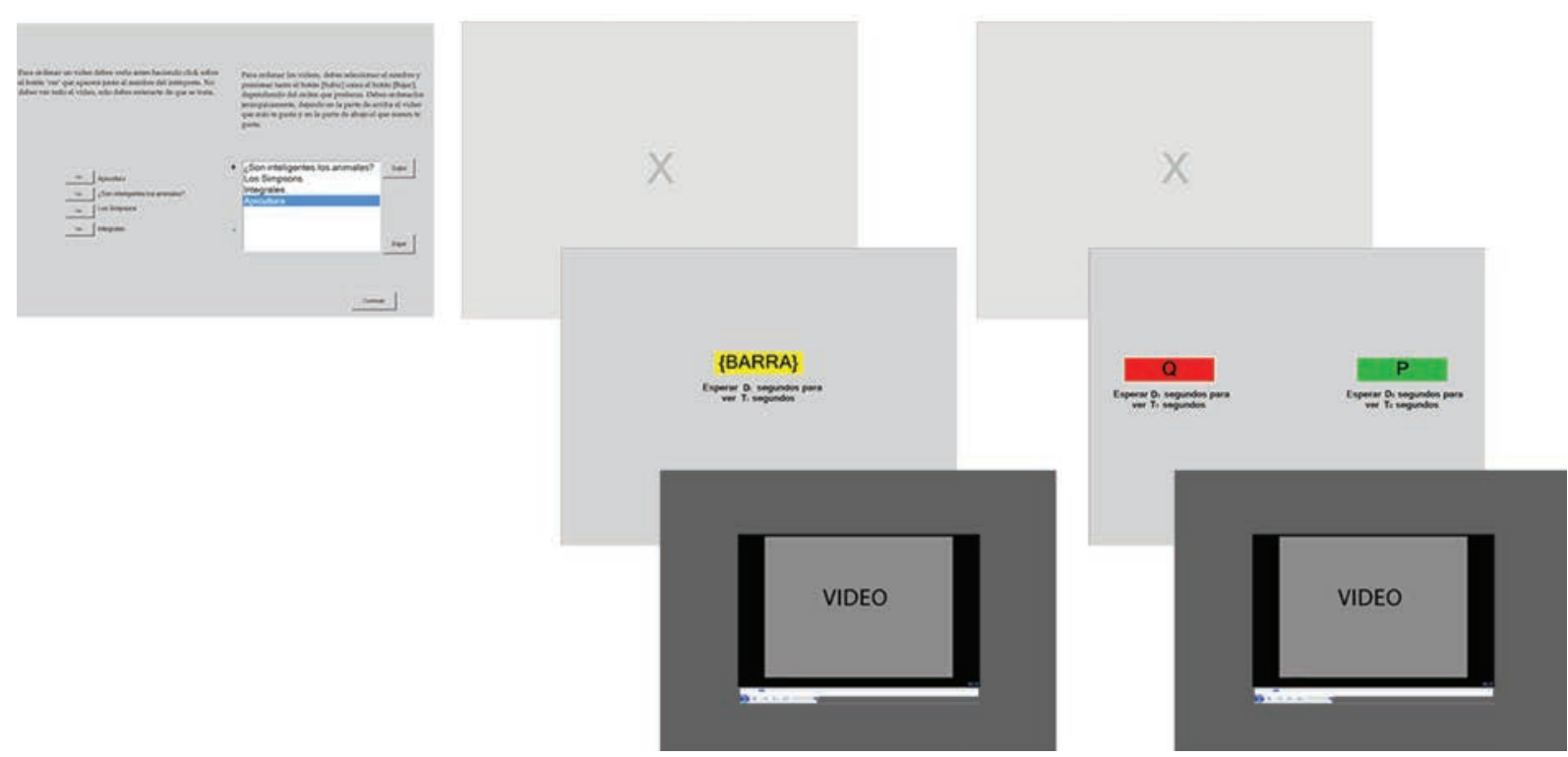

Figura 1. Descripción del procedimiento general y los bloques de ensayos 
en la tabla 1. De este modo, la manipulación de la magnitud estuvo dada por la duración de exposición al video, en tanto que la demora hizo referencia al tiempo desde el momento de la elección que los participantes debían esperar antes de que se presentara un fragmento del video. La exposición a cada tratamiento se realizaba en bloques de tres ensayos; los dos primeros ensayos fueron de no elección, de tal modo que se presentaba una de las dos alternativas del bloque de manera aislada, y el tercero de elección, presentándose simultáneamente ambas alternativas del bloque. Los ensayos de no elección fueron empleados con el fin de que los participantes se expusieran previamente a los valores de demora y tiempo de exposición asociados con las alternativas que serían presentadas en el ensayo de elección siguiente.

Con el fin de no extender más allá de una hora el tiempo de sesión, a los grupos que tenían más tratamientos definidos se les presentó un menor número de bloques de ensayos. Se asumió, por tanto, (Shapiro, Siller \& Kacelnik, 2008) que los valores particulares utilizados en cada tratamiento no tuvieron un efecto sobre la elección. Al presentarse un menor número de bloques en los grupos con mayor cantidad de tratamientos se igualó el número de ensayos de exposición a cada una de las razones programadas, lo que facilitó la comparación de la elección entre grupos.

Cada ensayo se inició con la presentación en el centro de la pantalla, por un periodo de 2 segundos, de la letra "X". Posteriormente, en el caso de los ensayos de no elección, en el centro de la pantalla aparecería la etiqueta "espacio", y debajo de ella se mostraban tanto la demora como el tiempo de exposición al video de la siguiente manera: "Esperar X segundos, para ver Y segundos". Si se trataba de un ensayo de elección, al lado izquierdo y derecho de la pantalla, aparecían las etiquetas [Q] y [P], respectivamente, y debajo de cada una de ellas se especificaba la demora y tiempo de exposición de la misma manera que en los ensayos de no-elección. En las instrucciones de la tarea se señaló que el sujeto debía presionar en el teclado

Tabla 1

Valores de tiempo de exposición-demora de las alternativas utilizadas en cada tratamiento para cada grupo

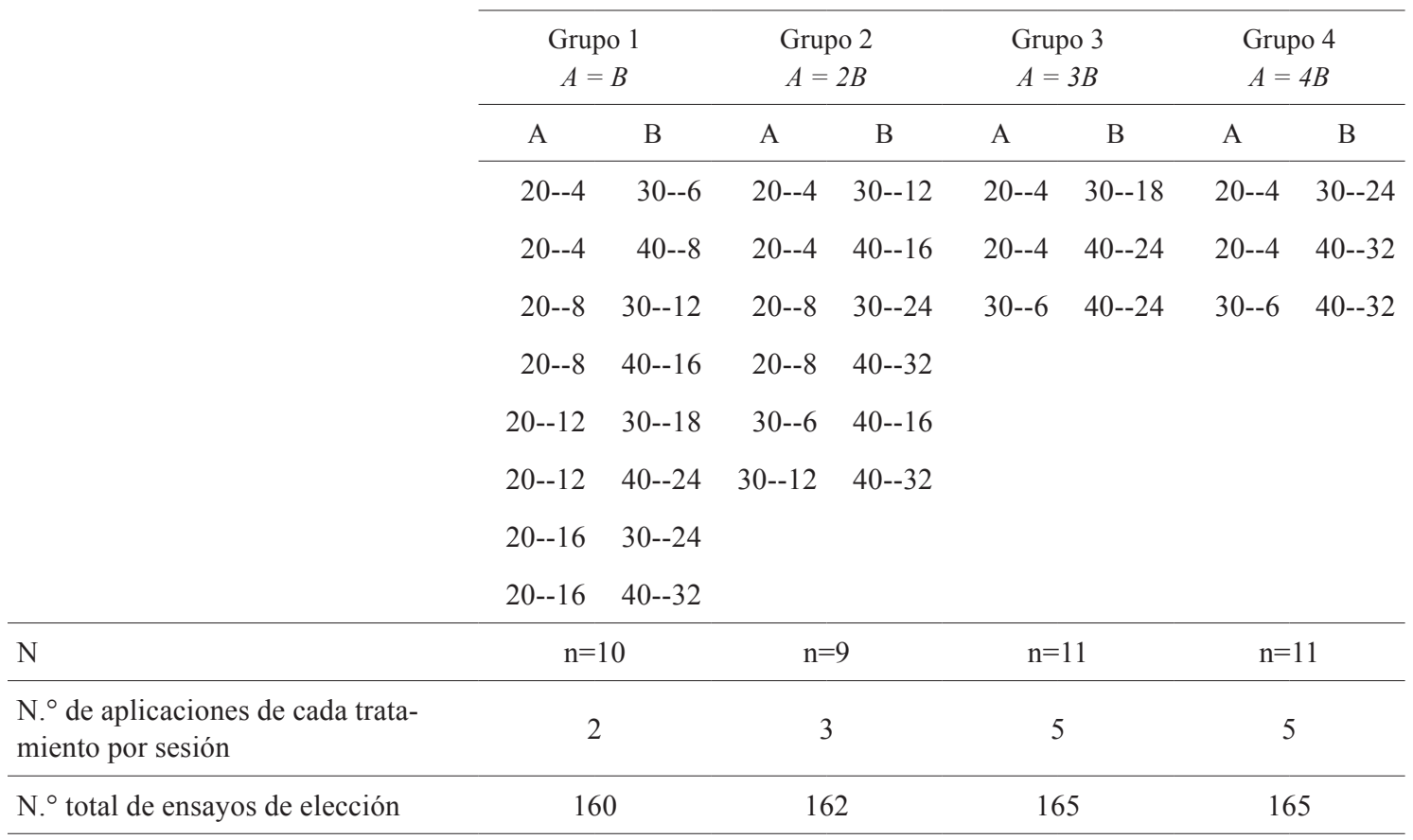

Nota: (T-D): $\mathrm{T}=$ tiempo de exposición al video; $\mathrm{D}=$ demora programada. 
de la computadora las teclas correspondientes a las etiquetas que aparecían en pantalla en cada ensayo según fuera el caso. A saber, [Q], [P] o [barra espaciadora].

Se aleatorizó la posición en pantalla en la que se presentaron las alternativas A y B. En ambos tipo s de ensayos, tras la elección del participante, se eliminaban las alternativas de la pantalla e iniciaba la demora programada. Finalizada la demora, daba inicio la presentación del segmento de video correspondiente a la alternativa elegida. El segmento presentado era continuación del fragmento de video que había sido presentado en el segmento inmediatamente anterior. Se programó un intervalo entre ensayos de 5 segundos de duración. La duración de los videos no permitía que estos pudieran finalizar antes de concluir la sesión experimental.

\section{Resultados}

Los resultados se analizan considerando la preferencia y ejecución en cada uno de los grupos, sin considerar la diferencia entre tratamientos. No se reportan análisis individuales de los tratamientos de cada uno de los grupos.

Se calculó la probabilidad de elegir la alternativa A para cada grupo dividiendo el número de bloques donde fue elegida en los ensayos de elección libre por el número total de bloques (figura 2). La preferencia por la alternativa A incrementó a medida que se aumentaba la diferencia entre la razones T-D en ambas alternativas de respuesta. Contrario a lo esperado en el grupo $1(\mathrm{~A}=\mathrm{B})$, en el que ambas alternativas de respuesta entregaban la misma razón T-D, la probabilidad de elección de la alternativa A no fue cercana a 0,5 , observándose un sesgo o preferencia por la alternativa B ( $\chi 2(1)$ $=7,316 ; \mathrm{p}<0,01)$, aquella que entregaba en todos los tratamientos tanto tiempos de exposición mayores, como demoras más altas. En el grupo $2(\mathrm{~A}=$ 2B) no se observaron diferencias estadísticamente significativas en la preferencia por ambas alternativas, si bien los resultados encontrados fueron muy cercanos a la significatividad estadística $(\chi 2(1)$ $=3,556: \mathrm{p}=0,059)$, en tanto que en los grupos 3 $(\mathrm{A}=3 \mathrm{~B})$ y $4(\mathrm{~A}=4 \mathrm{~B})$ se observó preferencia por la alternativa $A(G 3: \chi 2(1)=13,388: p<0,01 ; G 4$ : $\chi 2(1)=6,6: p=0,01)$. Estos datos muestran que los participantes fueron sensibles a la diferencia de razones T-D programadas en cada uno de los grupos. La preferencia observada en el grupo 1 señala un sesgo por la alternativa $\mathrm{B}$, aquella alternativa que daba acceso en todos los casos a un mayor tiempo de exposición al video (tabla 1), si bien requería una demora más alta (sobre este punto se regresará en la discusión). Si asumimos un sesgo en todos los grupos por la alternativa B, los resultados obtenidos muestran que al incrementar la diferencia de razones entre grupos aumentó la probabilidad de elección de la alternativa que daba acceso a un mayor valor de la razón T-D, hasta alcanzar un nivel asintótico determinado por el sesgo por la alternativa $\mathrm{B}$, de tal suerte que la preferencia por la alternativa A no aumentó cuando se incrementó en más de 3 veces la razón T-D a la que daba acceso, en relación con la alternativa B. Estos datos proporcionan evidencia a favor de la razón T-D como una medida del valor atribuido a cada una de las alternativas.

Se analizaron de manera independiente las latencias de respuesta para los ensayos de no-elección y elección. En la figura 3 se muestra la mediana de las latencias de respuesta (en décimas de segundo) para las alternativas A y B, para cada uno de los grupos en los ensayos de no-elección y elección. No se observaron diferencias en las latencias de respuesta en los ensayos de no elección, ni entre grupos ni entre las alternativas de respuesta para cada uno de los grupos. De manera consistente, las latencias de respuesta se incrementaron en los cuatro grupos en los ensayos de elección, siendo el incremento mayor para las alternativas B de respuesta que para las alternativas $\mathrm{A}$. 


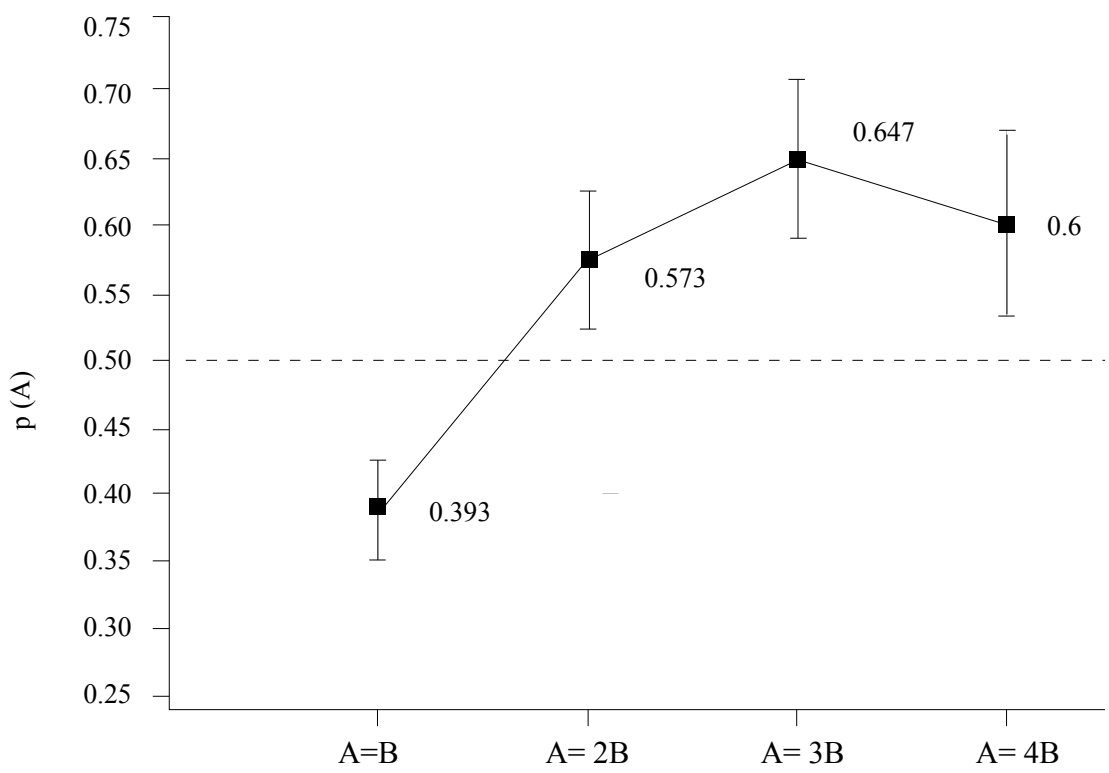

Figura 2. Probabilidad promedio $( \pm \mathrm{SEM})$ de elegir alternativa A por grupo en ensayos de elección

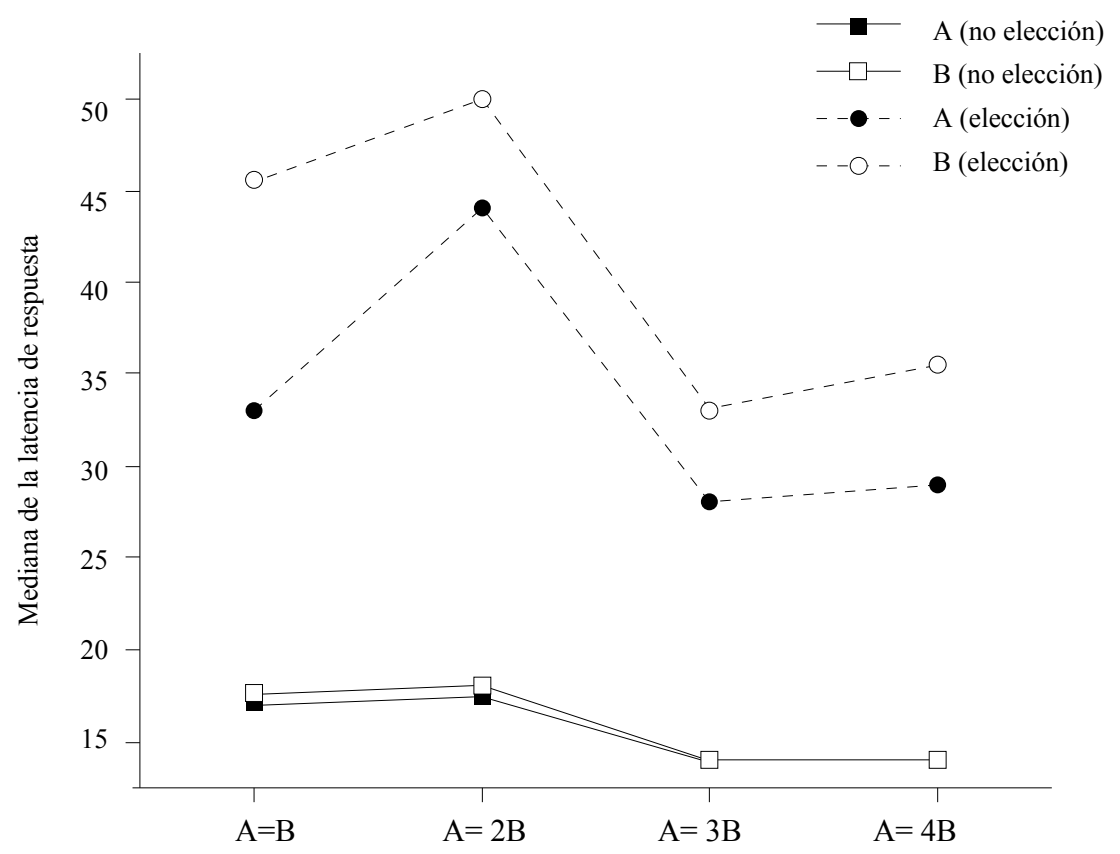

Figura 3. Medianas de latencia de respuesta (en décimas de segundo) correspondiente a cada grupo

Nota: Las líneas continuas y discontinuas representan los ensayos de no-elección y elección respectivamente. Las figuras llenas hacen referencia a la alternativa $\mathrm{A}$ en tanto que las huecas representan la alternativa $\mathrm{B}$. 
Se analizó el efecto del grupo (incremento de la diferencia en la razón tiempo de exposición-demora) sobre la latencia de respuesta. En la figura 4 se grafican los incrementos relativos de las medianas de las latencias de respuesta por alternativa y grupo entre los ensayos de no elección y los de elección; para ello se dividió la latencia promedio de los ensayos de elección por la correspondiente a los ensayos de no-elección para cada una de las alternativas y grupos.

La figura 4 muestra que las latencias de respuesta incrementaron de los ensayos de no-elección a los de elección en todos los grupos. Las latencias incrementaron en mayor medida para la alternativa $\mathrm{B}$ que para la alternativa A; particularmente, fueron 2,13 veces mayores en el caso de la alternativa $A$ y 2,57 veces más altas para la alternativa $\mathrm{B}$. Al comparan los incrementos en las latencias entre grupos, se observa que estos ocurrieron proporcionalmente para ambas alternativas en función de las diferencias entre las razones T-D programadas en cada uno de los grupos, si bien la correlación entre los incrementos de latencias para las alternativas A y B no resultó significativa ( $r h o=0,4 ; p$ $=0,6$ ) al estimarse la correlación con únicamente 4 valores por alternativa de respuesta.

Con la finalidad de contrastar si la latencia observada en los ensayos de no-elección de cada uno de los bloques era predictora de la elección posterior en el ensayo de elección libre, se calculó la proporción de bloques en los que la latencia en un ensayo de no-elección correspondiente a la alternativa A fue menor que la latencia evocada por la alternativa B. Esta proporción fue contrastada con la proporción de elecciones de la alternativa A en ensayos de elección libre. Los resultados se muestran en la figura 5.

Para cada tratamiento se realizó un análisis de regresión lineal. Se obtuvieron coeficientes de determinación por encima de 0,8 excepto para el grupo $1(\mathrm{~A}=\mathrm{B})$. Se compararon las pendientes obtenidas con las esperadas. Únicamente se observaron diferencias estadísticamente significativas entre las rectas de regresión estimadas considerando los

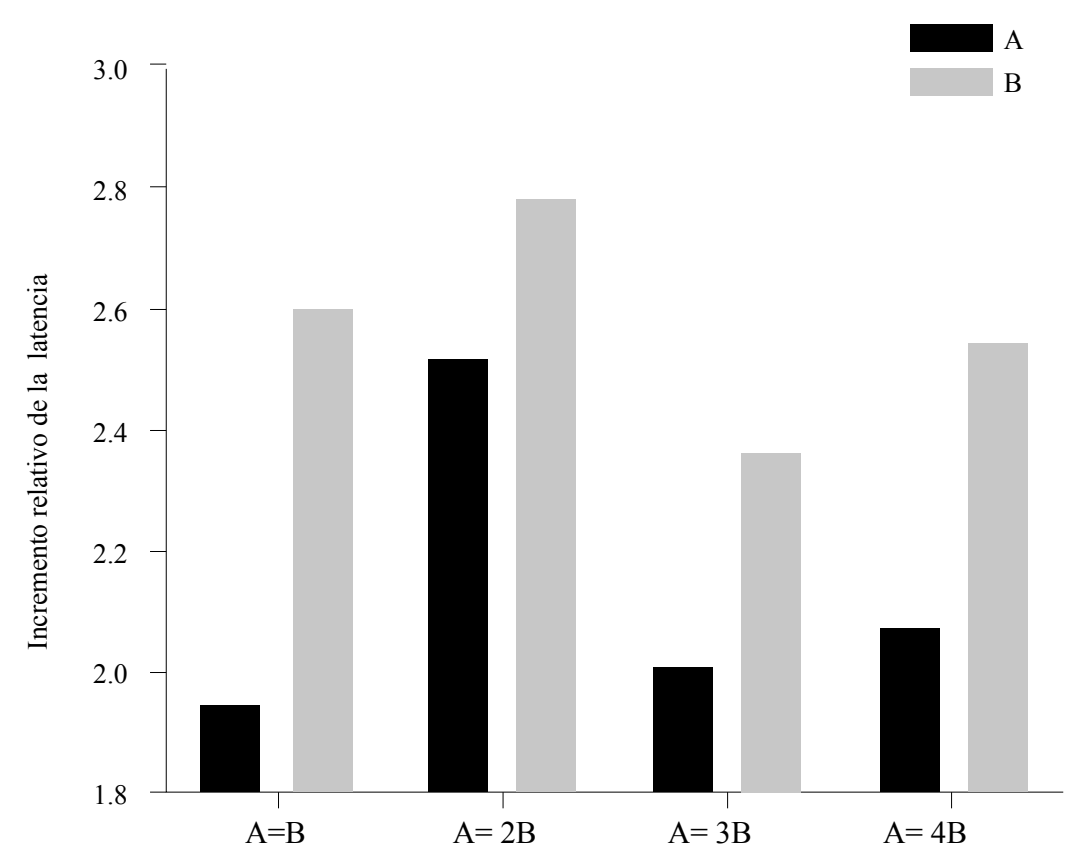

Figura 4. Incremento relativo observado en las latencias entre los ensayos de no-elección y los de elección para cada uno de los grupos

Nota: En negro se grafican los incrementos correspondientes a la alternativa A y en gris los de la alternativa B. 
supuestos del Modelo de Elección Secuencial y las estimadas a partir de las puntuaciones obtenidas para el grupo $2(\mathrm{~A}=2 \mathrm{~B} ; \mathrm{t}(14)=2,19 ; \mathrm{p}=0,046)$. Para los otros tres grupos no se observaron diferencias $(\mathrm{G} 1: \mathrm{t}(16)=1,57 ; \mathrm{p}=0,13 ; \mathrm{G} 3: \mathrm{t}(18)=$ $-0,1684 ; p=0,87 ; G 4: t(18)=-0,58 ; p=0,57)$. Estos datos permiten concluir que la latencia evocada por cada alternativa en los ensayos de no elección inmediatamente anteriores a un ensayo de elección fue un buen predictor de la preferencia posterior cuando ambas alternativas se presentaron de manera simultánea, especialmente para los grupos 3 y 4 , en los que era mayor la diferencia entre las razones T-D. Para los cuatro grupos, la relación entre la proporción de ensayos de no-elección en los que la latencia correspondiente a la alternativa A fue menor que la de la alternativa $\mathrm{B}$ y la elección posterior de la alternativa $\mathrm{A}$ en los ensayos de elección fue inferior a la esperada, de tal suerte que los participantes subigualaron.

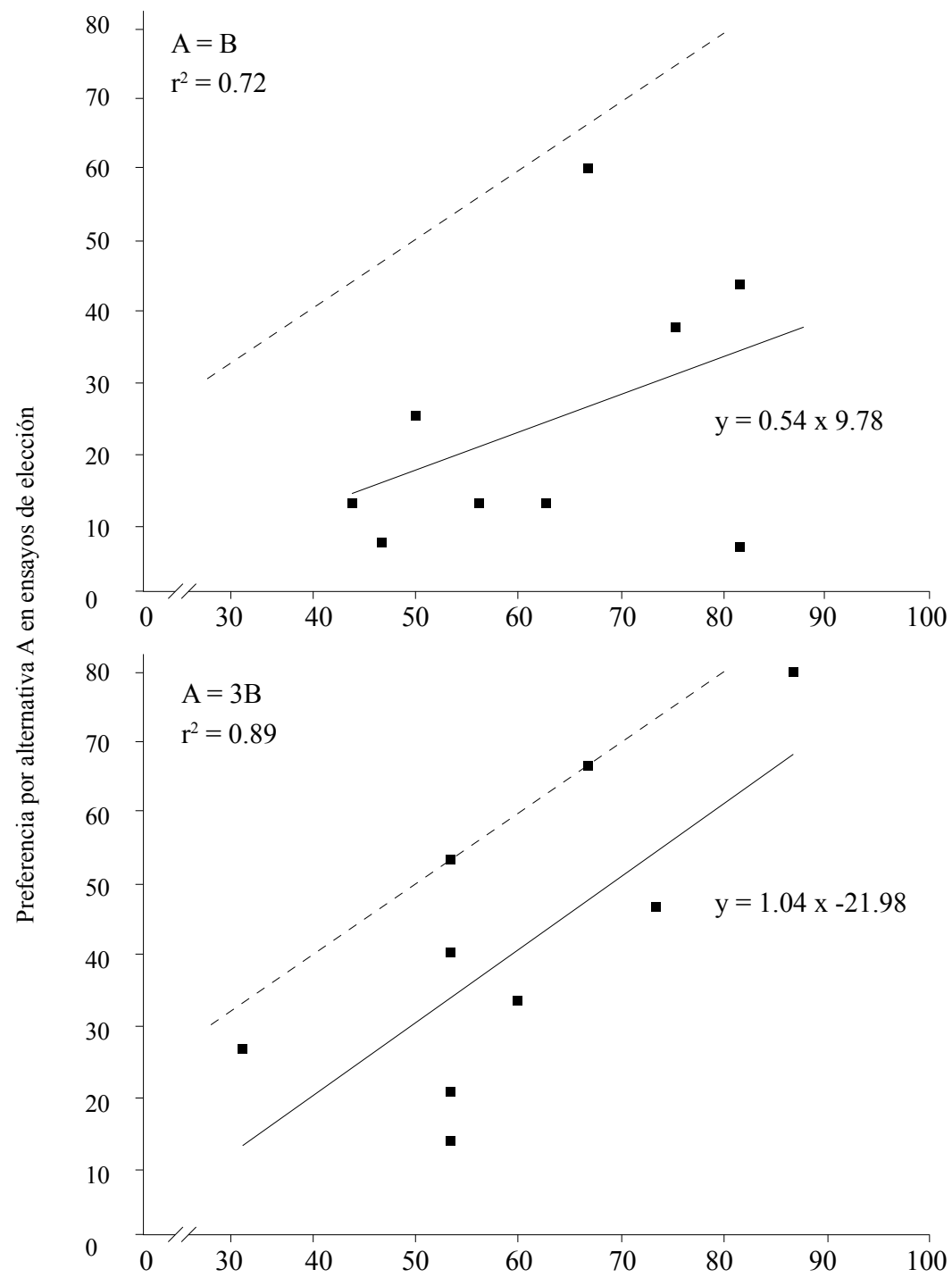

Proporción de bloques con latencias menores en A que en B (ensayos de no elección) 


\section{Óscar García-Leal, Enzo Leandro Rodríguez macías}

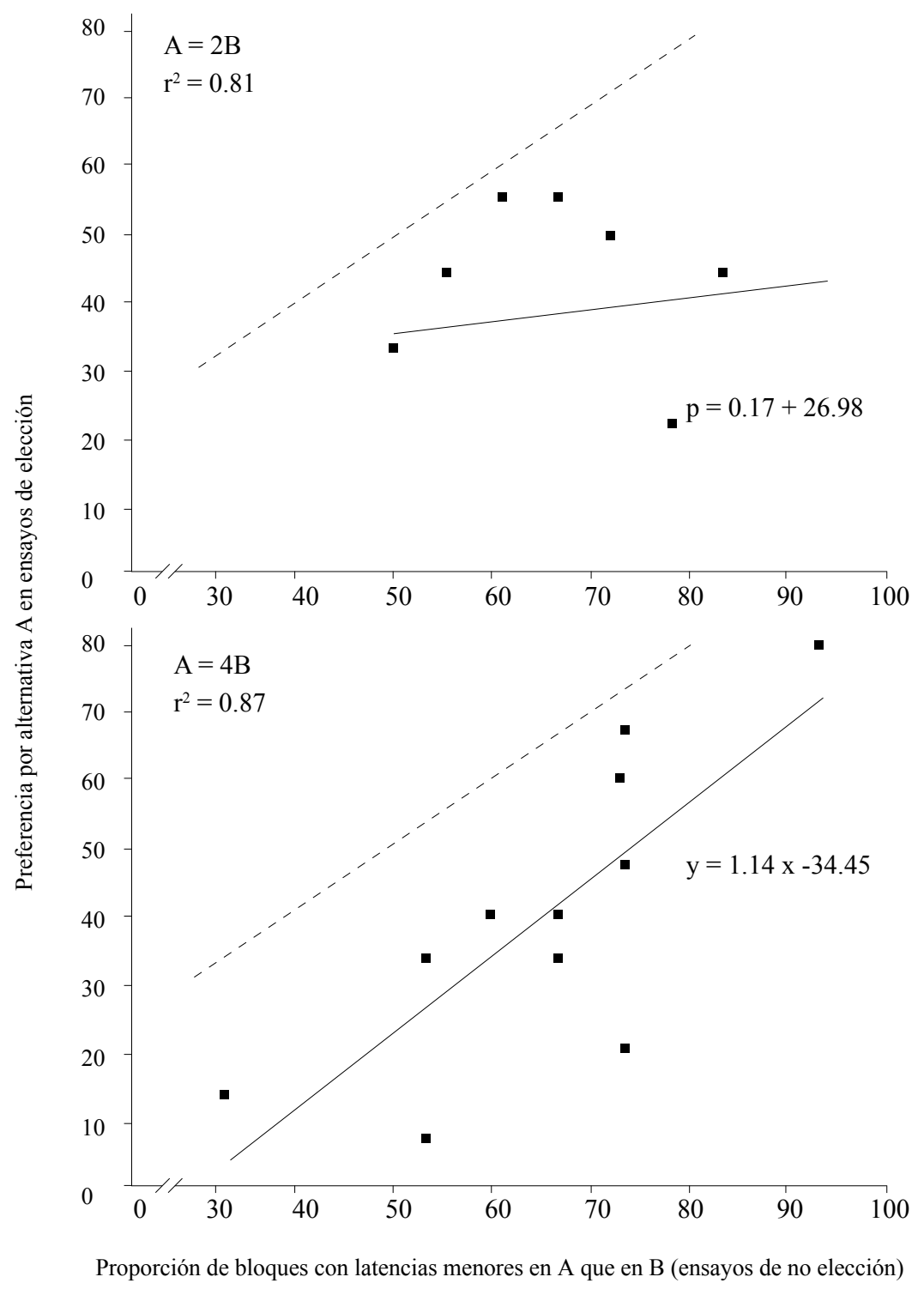

Figura 5. Proporción de bloques donde la latencia evocada por la alternativa A en ensayos de no-elección fue menor que la evocada por la alternativa B, comparado con la proporción de elecciones de A en ensayos de elección

Nota: Cada punto, dentro de cada grupo, representa la puntuación de un participante en toda la sesión, sin distinguir el tratamiento en particular al cual pertenecieron. Se presentan tanto la recta de regresión correspondiente a las puntuaciones observadas (línea continua) como a las esperadas (línea discontinua).

\section{Discusión}

Los resultados obtenidos apoyan parcialmente los supuestos del SCM, propuesto como una alternativa a los modelos de decisión que asumen un mecanismo básico de comparación de los valores de las alternativas que conforman el contexto de elección.

El SCM asume que los procesos implicados en la toma de decisiones cuando se presentan dos o más alternativas no difieren de los que operan cuando cualquiera de ellas se presenta de manera aislada. 
El SCM asume que dada la presentación de una alternativa en situaciones de no elección el tiempo de respuesta o latencia es una medida de con qué tanta fuerza esta alternativa evoca una respuesta hacia ella. En la medida en la que el valor atribuido por el organismo a la alternativa sea mayor, el drive o impulso para responder será también mayor, observándose una menor latencia de respuesta. De manera opuesta, la latencia será mayor cuanto menor sea el valor atribuido a la alternativa. De este modo, si se presenta una alternativa de respuesta de manera aislada de manera repetida y se registran las latencias de respuesta, será posible estimar una distribución de probabilidad particular de las latencias evocadas por cada una de las alternativas de respuesta.

Cuando se presentan dos o más alternativas el proceso es semejante al descrito, de tal modo que cada opción de respuesta evocará una latencia por responder. El valor evocado por cada una de las alternativas podrá ser estimado a partir de la distribución de probabilidad correspondiente a las latencias de respuesta observadas para cada alternativa en los ensayos de no elección. La alternativa elegida será aquella que evoque una latencia de respuesta más corta. De este modo, las alternativas presentadas competirán por ser expresadas en la elección. Este mecanismo no requiere por tanto aludir a procesos de comparación del valor de las alternativas.

Específicamente, el modelo establece las siguientes predicciones: (i) el proceso de valuación involucra aprendizaje y refleja el valor de la opción presente en relación al resto de las alternativas de respuesta, (ii) en general, se observarán latencias menores en los ensayos de elección que en los ensayos de no-elección, particularmente, cuando se elija la alternativa de menor valor atribuido y (iii) como resultado, se predicen preferencias parciales entre las alternativas en ensayos de elección libre a partir de las latencias observadas en los ensayos inmediatamente anteriores de no-elección (Kacelnik et al., 2001).
Hasta el momento los estudios que han abordado la validación del SCM se han restringido a estudios con animales. El objetivo de este trabajo fue ampliar estos estudios utilizando humanos como sujetos experimentales, bajo el supuesto de la existencia de mecanismos análogos de elección en animales y humanos. Una de las hipótesis propuestas plantea que la diferencia en la tendencia de elección entre humanos y otros animales estriba en diferencias en el tipo de recompensa entregada. Particularmente, asume que en tanto que en tareas diseñadas para el estudio de elección en animales no humanos la consecuencia entregada es un consumible (i.e., comida, agua...) y por tanto su efecto es inmediato, en el caso de las tareas con humanos las consecuencias entregadas no tienen este valor (i.e., puntos posteriormente intercambiables por recompensas, créditos en materias...).

En la figura 2 se muestra la preferencia por la alternativa A en ensayos de elección para cada uno de los grupos considerados. Si bien, no se observó indiferencia para el grupo 1, en el que la razón T-D se igualó para ambas alternativas, sino una probabilidad de elección mayor de la alternativa $B$ (asociada con un mayor valor de T), sí se observó un incremento en la probabilidad de elección de A al incrementarse la diferencia en la razón T-D entre ambas alternativas, hasta alcanzar un nivel asintótico. Este dato, a juicio de esta investigación, permite afirmar que los participantes fueron (i) sensibles a las diferencias en las razones T-D y (ii) proponer un mayor peso del tiempo de exposición al video que de la demora hasta su inicio. La interpretación de los resultados obtenidos, se realizará, por tanto, asumiendo que la razón T-D es una medida adecuada del valor atribuido a las alternativas de respuesta empleadas en este estudio. El efecto de esta misma razón, pero en esta ocasión entre magnitud de recompensa y demora a esta, ha sido reportado con animales (Shapiro et al., 2008). Este resultado sugiere que el empleo de recompensas consumibles dentro de la tarea pudiera propiciar una tendencia de elección similar 
(Locey et al., 2009) entre animales no humanos y humanos, si bien algunos estudios han reportado tendencias de elección similares cuando se utilizan elecciones hipotéticas en comparación con el uso de reforzadores no consumibles inmediatamente (Green, Myerson, Lichtman, Rosen \& Fry, 1996).

Los datos obtenidos en relación con la latencia y la preferencia fueron consistentes con la primera de las predicciones empíricas enunciadas del SCM, observándose latencias menores en todos los grupos para la alternativa A que para la B. En todos los casos, la alternativa A era aquella en la que la consecuencia se asociaba tanto con una demora como con un tiempo menor de exposición al video. Este hallazgo es consistente con los encontrados en otros estudios donde se analizan otros procesos de decisión en animales como: elección bajo riesgo (Aw et al., 2012; Craft, 2016), elección racional (Schuck-Paim \& Kacelnik, 2002) y sesgo pesimista (Mendl, et al., 2010). En dichos estudios las latencias más cortas estuvieron asociadas con la alternativa preferida. Esta relación entre latencias cortas y la alternativa preferida también ha sido documentada en estudios en mercadotecnia en humanos (Aaker, Bagozzi, Carman \& MacLachlan, 1980). Sin embargo, dentro de esta misma área también se ha observado la tendencia inversa. Por ejemplo, Reimann, Castaño, Zaichkowsky \& Bechara (2012) encontraron latencias de elección más grandes hacia logotipos nuevos y preferidos. Es posible que esta inconsistencia sea debida a que los sujetos responden de manera diferente ante alternativas novedosas que ante alternativas preferidas. Este efecto ha sido reportado en estudios donde se analiza la latencia hacia estímulos incompatibles (Alluisi, Strain \& Thurmond, 1964) y situaciones de conflicto entre estímulos (Gonzalez-Rosa et al., 2013), condiciones que provocan incremento en las latencias.

En relación con la predicción (ii) del SCM los datos no muestran latencias menores en los ensayos de elección libre que en los ensayos de no-elección (figuras 3 y 4). Los datos muestran un incremento en las medianas de las latencias en los ensayos de elección en relación con las observadas en los ensayos de no-elección. Este incremento fue mayor para la alternativa de menor valor atribuido, esto es, la alternativa B. Asimismo, el incremento observado afectó del mismo modo a las alternativas A y B en función del grupo. Otros estudios con animales han mostrado evidencia inconsistente con esta predicción. Por ejemplo, Roche et al. (1997), empleando un procedimiento de sensibilidad al riesgo, reportaron latencias iguales en ensayos de elección y no elección, o bien latencias mayores en ensayos de elección. Las latencias mayores observadas en los ensayos de elección sugieren la existencia de un costo de valoración que es función del número de alternativas presentadas simultáneamente. Un mecanismo similar ha sido sugerido en estudios con humanos que analizan el efecto denominado paradoja de la elección (DarNimrod, Rawn, Lehman \& Schwartz, 2009), donde la presencia de más alternativas implica un costo para los sujetos, de tal suerte que incrementar el número de alternativas disminuyen los valores reportados de satisfacción en relación a la opción elegida. En animales, aumentar el número de alternativas que conforman el contexto de elección ha mostrado afectar el número de elecciones óptimas (Hutchinson, 2005). Sin embargo, son pocos los estudios donde se ha evaluado en animales el efecto de incrementar el número de alternativas sobre la latencia de elección.

Considerando lo anterior, es posible que otros mecanismos afecten las latencias durante los ensayos de elección y no elección en animales (e. g., costo de valoración o restricciones cognitivas), pero en el caso de este experimento no es posible establecer con precisión cuál de ellos tuvo efecto.

La última predicción fue evaluada utilizando el mismo procedimiento reportado en Shapiro et al. (2008). Los datos observados son consistentes con el SCM. Como se observa en la figura 5, se obtuvieron correlaciones significativas entre 
la proporción de bloques en los que la latencia correspondiente a la alternativa A en los ensayos de no-elección fue menor que la observada en la alternativa $\mathrm{B}$ y la elección posterior en el ensayo de elección libre inmediatamente posterior. Este hallazgo es consistente con otros estudios con animales (Aw et al., 2012; Shapiro et al., 2008)

En resumen, los resultados obtenidos muestran que las predicciones del SCM se observaron parcialmente en un estudio con humanos, cuando el procedimiento empleado se asemejó al utilizado con animales no humanos. Si bien, no se observó un decremento en las latencias de respuesta en los ensayos de elección en relación con la observada en los de no-elección, las latencias de respuesta en los ensayos de no-elección de las alternativas A y B fueron predictoras de la preferencia posterior. Estos datos contribuyen a la hipótesis de que los procesos implicados en la toma de decisiones en animales no humanos y humanos son comparables, así como que las variaciones en los procedimientos experimentales utilizados entre especies pudieran estar dando razón de las diferencias encontradas. Sin embargo, los datos no permiten descartar la hipótesis de la existencia de un mecanismo de comparación entre el valor de las alternativas en situaciones de elección libre.

\section{Referencias}

Aaker, D. A., Bagozzi, R. P., Carman, J. M., \& MacLachlan, J. M. (1980). On using Response latency to measure preference. Journal of Marketing Research, XVII, 237-244. doi:10.2307/3150934

Alluisi, E. A., Strain, G. S., \& Thurmond, J. B. (1964). Stimulus-response compatibility and the rate of gain of information. Psychonomic Science, 1 (1), 111-112. doi:10.3758/BF03342816

Aw, J., Monteiro, T., Vasconcelos, M., \& Kacelnik, A. (2012). Cognitive mechanisms of risky choice: Is there an evaluation cost? Behavioral Processes, 89, 95-103. doi:10.1016/j.beproc.2011.09.007
Bradshaw, C. M., \& Szabadi, E. (1992). Choice between delayed reinforcers in discrete-trials schedule: The effect of deprivation level. The Quarterly of Experimental Psychology, 44B(1), 1-16.

Craft, B. B. (2016). Risk-sensitive foraging: changes in choice due to reward quality and delay. Animal Behavior, 11, 41-47. doi:10.1016/j.anbehav.2015.09.030

Dar-Nimrod, I., Rawn, C. D., Lehman, D. R., \& Schwartz, B. (2009). The maximization Paradox: The cost of seeking alternatives. Personality and Individual Differences, 46, 631-635. doi:10.1016/j.paid.2009.01.007

Feeney, M. C., \& Roberts, W. A. (2008). Rats show preference for delayed rewards on the radial maze. Learning and Behavior, 36(1), 42-54. doi:10.3758/lb.36.1.42

Forzano, L. B., \& Logue, A. W. (1994). Self-control in adult humans: Comparison of qualitatively different reinforcers. Learning and Motivation, 25, 65-82. doi:10.1006/lmot.1994.1004

Freidin, E., Aw, J., \& Kacelnik, A. (2009). Sequential and simultaneous choices: Testing the diet selection and sequential choice models. Behavioural Processes, 80, 218-223. doi:10.1016/j.beproc.2008.12.001

Gonzalez-Rosa, J. J., Innuggi, A., Blasi, V., Cursi, M., Annovazzi, P., Comi, G. et al. (2013). Response competition and response inhibition during different choice-discrimination task: Evidence from ERP measured inside MRI scanner. International Journal of Psychophysiology, 89, 37-47. doi:10.1016/j.ijpsycho.2013.04.021

Green, L., Myerson, J., Lichtman, D., Rosen, S., \& Fry, A. (1996). Temporal Discounting in Choice Between Delayed Rewards: The Roles of Age and Income. Psychology and Aging, 11(1), 7984. doi:10.1037//0882-7974.11.1.79

Hutchinson, J. M. C. (2005). Is more choice always desirable? Evidence and arguments from le$\mathrm{ks}$, food selection, and environmental enrichment. Biological Reviews Cambridge Phi- 
losophical Society, 80, 73-92. doi:10.1017/ s1464793104006554

Kacelnik, A., Vaconcelos, M., Monteiro, T., \& Aw, J. (2011). Darwin's "tug-of-war" vs. starlings' "horse-racing": How adaptations for sequential encounters drive simultaneous choice. Behavioral Ecology and Sociobiology, 65, 547-558. doi:10.1007/s00265-010-1101-2

Kahneman, D., \& Tversky, A. (1979). Prospect theory: An analysis of decision under risk. Econometrica, 47(2), 263-291. doi:10.2307/1914185

Kollins, S. H. (2003). Delay discounting is associated with substance use in college students. Addictive Behavior, 28, 1167-1173. doi:10.1016/S03064603(02)00220-4

Locey, M., Hackenberg, T., \& Pietras, C. (2009). Human risky choice: Delay sensitivity depends on reinforcer type. Journal of Experimental Psychology, 35(1), 15-22. doi:10.1037/a0012378

Logue, A. W., Peña-Correal, T. E., Rodriguez, M. L., \& Kabela, E. (1986). Self-control in adult humans: Variation in positive reinforcer amount and delay. Journal of the Experimental Analysis of Behavior, 46, 159-173. doi:10.1901/ jeab.1986.46-159

Luce, R. D. (1986). Response Times. New York: Oxford University Press.

Mazur, J. E. (2010). Distributed versus exclusive preference in discrete-trial choice. Journal of Experimental Psychology: Animal Behavior Processes 36, 321-333. doi:10.1037/a0017588

Mendl, M., Brooks, J., Basse, C., Burman, O., Paul, E., Blackwell, E., \& Casey, R. (2010). Dogs showing separation-related behavior exhibit a 'pesimistic' cognitive bias. Current Biology, 20(19), R839-R840. doi:10.1016/j. cub.2010.08.030

Mitchell, S. H. (2011). The genetic basis of delay discounting and its genetic relationship to alcohol dependence. Behavioral Processes, 87, 10-17. doi:10.1016/j.beproc.2011.02.008

Navarick, D. J. (1996). Choice in humans: Techniques for enhancing sensitivity to reinforcement immediacy. The Psychological Record, 46, 539554.

Navarick, D. J. (1998). Impulsive choice in adults: How consistent are individual differences? The Psychological Record, 48, 665-674.

Navarick, D. (2004). Discounting of delayed reinforcers: Measurements by questionnaires versus operant choice procedures. The Psychological Record, 54, 85-94.

Pietras, C., \& Hackenberg, T. (2003). Human risky choice across four probability distributions. The Psychological Record, 53, 443-457.

Reimann, M., Castaño, R., Zaichkowsky, J., \& Bechara, A. (2012). Novel versus familiar brands: An analysis of neurophysiology, response latency, and choice. Marketing letters, 23, 745-759. doi:10.1007/s11002-012-9176-3

Roche, J. P., Timberlake, W., \& McCloud, C. (1997). Sensitivity to variability in food amount: risk aversion is seen discrete-choice, but not in free-choice, trials. Behaviour, 134, 1259-1272. doi:10.1163/156853997x00142

Schuck-Paim, C., \& Kacelnik, A. (2002). Rationality in risk sensitive foraging choices by starlings. Animal Behaviour, 64, 869-879. doi:10.1006/ anbe. 2003.2003

Shapiro, M.S., Siller, S., \& Kacelnik, A. (2008). Simultaneous and sequential choice as a function of reward delay and magnitude: Normative, descriptive and process-based models tested in European starling (Sturnus vulgaris). Journal of Experimental Psychology: Animal Behavior Processes, 34, 75-93. doi:10.1037/00977403.34.1.75

Sigman, M., \& Dehaene, S. (2011). Why does it take time to make a decision? The role of a global workspace in simple decision making. En: O. Vartain y D. Mandel (Eds.), Neuroscience of Decision Making (pp. 11-44). New York: Psychology Press.

Tanno, T., Kurashima, R., \& Watanabe, S. (2011). Motivational control of impulsive behavior interacts with Choice opportunities. Learning 
and Motivation, 42, 145-153. doi:10.1016/j. lmot.2011.01.001

Tversky, A., \& Kahneman, D. (1981). The framing of decision and the psychology of choice. Science, 211, 453-458.

Recibido: Mayo 10, 2016

fprobado: Enero 21, 2017
Vasconcelos, M., Monteiro, T., Aw, J., \& Kacelnik, A. (2010). Choice in multi-alternative environments: A trial by trial implementation of the sequential choice model. Behavioural Processes, 84, 435-439. doi:10.1016/j.beproc.2009.11.010 
